\title{
Handbuch
}

\author{
der \\ staatlichen Denkmalpflege \\ in \\ Elsafs-Lothringen.
}

Im Auftrage des Kaiserlichen Ministeriums

für Elsaß-Lothringen

bearbeitet von

F. Wolff

Konservator der geschichtlichen Denkmäler im Elsaß.

Straßburg.

Verlag von Karl J. Trübner.

1903. 



\section{Zur Ur- und Frühgeschichte}

Elsass- Lothringen

R. FORRER.

Groß $4^{0}$. 46 Seiten Text nebst vor- und frühgeschichtlicher Fundtafel mit. 192 Abbildungen in Licht- und Farbendruck, im Format $65 \times 85 \mathrm{~cm}$. 1901. Mk. 3.-

Nach dem Vorbild anderer Staaten (Württemberg, preuß. Provinz Sachsen, Österreich u. a.) erscheint hiermit auch für Elsaß-Lothringen eine gemeinverständliche Aufklärung über die ur- und frühgeschichtlichen einheimischen Denkmäler. In dem Maße, wie die prähistorische Wissenschaft auch in Deutschland endlich nach und nach zu ihrem Rechte gelangt, ist es wichtig, daß dem Verschleudern und Verschleppen neuer Funde vorgebeugt und dem Volke die Anmeldepflicht besser als bisher nahegelegt werde. Dies kann nicht allein durch schriftliche Belehrung erfolgen; die bildliche Anschauung muß damit Hand in Hand gehen. Der Verfasser hat nun eine Fundtafel zusammengestellt, die alle bisherigen an Vollständigkeit und Übersichtlichkeit übertrifft und dieser Tafel einen Text beigefügt, der sowohl des Verfassers methodologische Ansichten auf dem Gebiete der Prähistorie enthält, wie auch eine in großen Zügen gehaltene Urgeschichte Elsaß-Lothringens und der angrenzenden Länder.

\section{WÖRTERBUCH DER ELSÄSSISCHEN MUNDARTEN BEARBEITET VON}

E. MARTIN und H. LIENHART.

IM AUFTRAGE DER LANDESVERWALTUNG VON ELSASS-LOTHRINGEN.

Erster Band. Lex. $-8^{\circ}$. XVI, 800 S. 1899 . Broschiert M. 20.-, in Halbfranz gebunden M. 22.50.

Der II. (Schluss-)Band ist in Vorbereitung. Er wird in etwa 5-6 Lieferungen à M. 4.- erscheinen.

c... Das elsässische Wörterbuch ist keine Aufspeicherung sprachwissenschaftlicher Raritäten. Es ist eine lebensvolle Darstellung dessen, wie das Volk spricht. In schlichten Sätzen, in Fragen und Antworten, in Anekdoten und Geschichtchen kommt der natürliche Gedankenkreis des Volkes zu unmittelbarer Geltung. Die Kinderspiele und die Freuden der Spinnstuben treten mit ihrem Formelapparat auf. Die Mehrzahl der Artikel spiegeln das eigentliche Volksleben wieder und gewähren dadurch einen wahren Genuss. Wenn man Artikel wie Esel oder Fuchs liest, wird man bald verstehen lernen, dass in deren Schlichtheit und Schmucklosigkeit der Erforscher deutschen Volkstums eine sehr wertvolle Quelle für das Elsass findet ... Strassburger Post 1897 Nr. 344. 


\section{Die Betmeindeorbunthy \\ für}

\section{Eliaßs-Pothringen vom 6. Iuni 1895

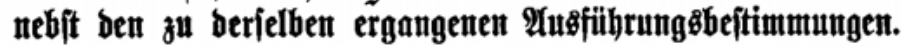

Bearbeitet bon

9. \$allen,

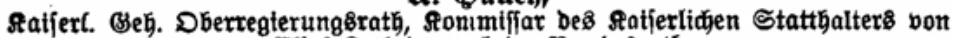

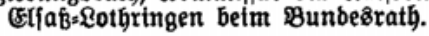

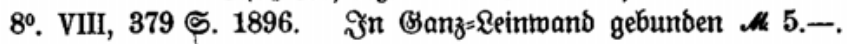

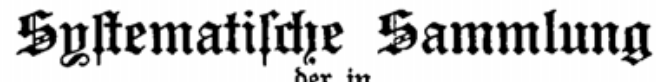

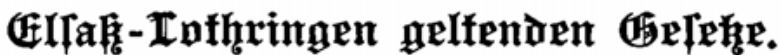

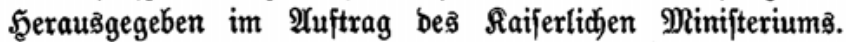

Eriter und zmeiter Band

Privatredt

bearbeitet von

Sarl \$affrath und శ̊ranz Brofimann,

Satjert. \&anogerichts̊răthe in @traß̄burg.

I. Band: Rer. $8^{\circ}$. XXII, 660 ธ. 1900 . Beheftet $\boldsymbol{M} 15 .-$, in foltbem Salbfranzband $\mathscr{A}$ 17.-.

II. Banb: \&er. $8^{\circ}$. XXXIV, 1200 S. 1901 . Bsebeftet Ak 24.50, in folidemn Đaltbranzband $\mathscr{A} 26.50$.

Die Einführung des Bürgerlichen Gesetzbuchs hat auch eine neue Bearbeitung der alten Möller'schen Gesetzsammlung nötig gemacht, da sie durch die neue Gesetzgebung und die damit verbundene Aufhebung einer großen Anzahl alter Gesetze nur noch in beschränktem Umfange gebrauchsfähig war. Auf Anweisung des Kaiserlichen Ministeriums erscheint die neue Ausgabe nicht mehr chronologisch, sondern systematisch nach den einzelnen Rechtsmaterien geordnet.

Die Sammlung wird mit dem Privatrecht erŏffnet, das in zwei Bänden vollständig vorliegt. Der erste Band enthält das Bürgerliche Gesetzbuch, das Gesetz über die Angelegenheiten der freiwilligen Gerichtsbarkeit, die Grundbuchordnung, das Handelsgesetzbuch, die Wechselordnung, das Gerichtsverfassungsgesetz, die Civilprozeßordnung, die Konkursordnung, das Gesetz über die Zwangsversteigerung und die Zwangsverwaltung, ferner die sämtlichen zu diesen Gesetzen gehörigen Ein- und Ausführungsgesetze, die Gerichtskostengesetze und die Gebührenordnungen: alles mit auf Elsaß-Lothringen bezüglichen kurzen Erläuterungen versehen; der zweite Band enthält in der gleichen Bearbeitung die sämtlichen übrigen Gesetze und Verordnungen privatrechtlichen Charakters bis auf die neueste Zeit.

Ein ausführliches Register am Schlusse des zweiten Bandes gewährt einen vollständigen und sicheren Überblick über den heutigen Rechtszustand. 

중 


\title{
Handbuch
}

\author{
der \\ staatlichen Denkmalpflege \\ in \\ Elsafs-Lothringen.
}

Im Auftrage des Kaiserlichen Ministeriums

für Elsaß-Lothringen

bearbeitet von

F. Wolff

Konservator der geschichtlichen Denkmäler im Elsaß.

Straßburg.

Verlag von Karl J. Trübner.

1903. 


\section{DuMont Schauberg, Strassburg.}

